\title{
POSITIVE DERIVATIONS ON $f$-RINGS
}

\author{
P. COLVILLE, G. DAVIS and K. KEIMEL
}

(Received 23 January 1976; revised 31 March 1976)

\section{Introduction}

Throughout this paper $A$ will denote an $f$-ring i.e. a lattice-ordered ring in the sense of Birkhoff and Pierce (1956) in which for all $x, y, z \in A, x \wedge y=0$ implies $x \wedge z y=0=x \wedge y z$.

A group endomorphism $D: A \rightarrow A$ is positive if $D(x) \geqq 0$ whenever $x \geqq 0$ in $A$. A derivation on $A$ is a group endomorphism $D: A \rightarrow A$ for which $D(x y)=x D(y)+D(x) y$ for all $x, y \in A$.

Our objective is to characterize algebraically the positive derivations on certain $f$-rings. Specifically, we show that if $A$ is an archimedean $f$-ring then the positive derivations on $A$ are precisely the positive endomorphisms of $A$ with range contained in the nilpotents of $A$ and vanishing on $A^{2}$.

\section{Derivations on archimedean $f$-rings}

We recall that $A$ is archimedean if for some $x, y \in A$ we have $n x \leqq y$ for all natural numbers $n$, then $x \leqq 0$. Birkhoff and Pierce (1956) have shown that every archimedean $f$-ring is commutative.

We denoted by $\operatorname{Rad}(A)$ the set of nilpotent elements of $A$. Birkhoff and Pierce (1956) show that $\operatorname{Rad}(A)$ is a convex sublattice and a two-sided ideal of $A$ (briefly, $\operatorname{Rad}(A)$ is an $l$-ideal) and that $A / \operatorname{Rad}(A)$ is a reduced ring that is, a ring with no non-zero nilpotents.

LEMMA 1. If $A$ is an archimedean $f$-ring then $R a d(A)$ is a polar subset of $A$. In particular, $A / R a d(A)$ is an archimedean $f$-ring.

Proof. We denote by $M$ the set of all $z \in A$ for which $|z| \leqq x y$ for some $x, y \in A$. Thus, $M$ contains all products and is an $l$-ideal of $A$. We let $M=\{x \in A:|x| \wedge|z|=0$ for all $z \in M\}$ be the polar of $M$. Then $M$ annihilates $A$ for if $a \in M$ and $b \in A$ then $a b \in M$ so we have $|a| \wedge|a b|=0$, and then $|a b|=|a b| \wedge|a b|=|a||b| \wedge|a b|=0$. Thus $M \subseteq \operatorname{Rad}(A)$. On the other 
hand, in the proof of their theorem 3.11 Henriksen and Isbell (1962) show that $\operatorname{Rad}(A) \cap M=(0)$ holds if $A$ is archimedean. Thus $\operatorname{Rad}(A) \subseteq M$ in this case, so $\operatorname{Rad}(A)=M$ is a polar subset and $A / \operatorname{Rad}(A)$ is archimedean by Bigard (1969).

LEMMA 2. Let $A$ be a commutative ring with characteristic 0 and $D: A \rightarrow A$ a derivation. If $a \in A$ is nilpotent then $D(a)$ is nilpotent.

Proof. Let $a$ be nilpotent in the commutative ring $A$ with characteristic 0 and let $D: A \rightarrow A$ be a derivation. We have $a^{n}=0$, for some natural number $n$, so $n a^{n-1} D(a)=0$ and therefore $a^{n-1} D(a)=0$. Now suppose that for some integer $k, 1 \leqq k \leqq n$, we have $a^{n-k} D(a)^{2 k-1}=0$. By applying $D$ to this expression and multiplying by $D(a)$ we get $a^{n-(k+1)} D(a)^{2(k+1)-1}=0$. We can therefore continue until $D(a)^{2 n-1}=0$, so $D(a)$ is nilpotent.

An endomorphism $T$ of the additive group of $A$ is a positive orthomorphism if $x \wedge y=0$ implies $x \wedge T(y)=0$ in $A$.

Theorem 3. (Bigard and Keimel (1969)). A positive orthomorphism of $A$ is a positive group endomorphism $T$ for which $T(M) \subseteq M$ for each minimal prime subgroup $M$ of $A$. If $A$ is archimedean and reduced (that is, without proper nilpotents) then a positive orthomorphism $T: A \rightarrow A$ is generalized translation i.e. $T$ satisfies $T(x y)=x T(y)$ for all $x, y \in A$.

LeмmA 4. If $D$ is a positive derivation on an archimedean reduced $f$-ring A then $D=0$.

Proof. We see firstly that $D$ is a positive orthomorphism. Suppose that $x \wedge y=0$ in $A$. We then have $x y=0$ so that $x D(y)+D(x) y=0$. Since $x, y \geqq 0$ and $D$ is positive we have $x D(y)=0=D(x) y$, and therefore $x \wedge D(y)=0$, since $A$ is reduced. Now by theorem $3, D$ is a generalized translation. Thus for all $x, y \in A$ we have both $D(x y)=x D(y)+D(x) y$ and $D(x y)=x D(y)$. That is, for all $x, y \in A$ we have $D(x) y=0$, so $D=0$, since $A$ is reduced.

We now prove the result mentioned in the introduction, algebraically characterizing positive derivations on archimedean $f$-rings. Notice that if $I \subseteq A$ is an ideal and $D: A \rightarrow A$ is a derivation then the map $\bar{D}: A / I \rightarrow A / I$ defined by $\bar{D}(a+I)=D(a)+I$ is a derivation.

Theorem 5. Suppose that $A$ is an archimedean $f$-ring. Then the positive derivations on $A$ are precisely the positive group endomorphisms $D: A \rightarrow A$ satisfying $D(A) \subseteq \operatorname{Rad}(A)$ and $D\left(A^{2}\right)=(0)$.

Proof. Let $A$ be archimedean and $D: A \rightarrow A$ a positive homomorphism. If $D$ is a derivation then $D(\operatorname{Rad}(A)) \subseteq \operatorname{Rad}(A)$ by lemma 2 , since $A$ 
is commutative, so we can define a positive derivation $\bar{D}$ of $A / \operatorname{Rad}(A)$ by $\bar{D}(x+\operatorname{Rad}(A))=D(x)+\operatorname{Rad}(A)$. By Lemma 1 and lemma 4 we then have $\bar{D}=0$. That is, $D(A) \subseteq \operatorname{Rad}(A)$. Since $\operatorname{Rad}(A)$ annihilates $A$, as we have noted in lemma 1 , we have $D(x y)=x D(y)+D(x) y=0$.

Conversely, suppose that $D(A) \subseteq \operatorname{Rad}(A)$ and $D\left(A^{2}\right)=(0)$. Then for all $x, y \in A$ we have $D(x y)=0=x D(y)+D(x) y$, so $D$ is a derivation.

\section{Bounded and almost-bounded elements}

The results of the previous section show that we cannot expect a positive derivation on an $f$-ring to be too far from being zero. In this section we pursue the idea that the kernel of a positive derivation must be large.

If $A$ has a multiplicative identity 1 then we say that $b \in A$ is bounded if $|b| \leqq n 1$ for some natural number $n$. We note that if $D: A \rightarrow A$ is a positive derivation then $D(b)=0$ for all bounded elements $b$ of $A$ since $D(1)=0$.

A subset $P$ of $A$ is a prime $l$-ideal if $P$ is a convex sublattice ideal of $A$ for which the set $\{a \in A: a \cdot \geqq 0, a \notin P\}$ is closed under finite meet. A minimal prime $l$-ideal is a prime $l$-ideal minimal in the family of all prime $l$-ideals of $A$, ordered by inclusion. A family $\left\{P_{\lambda}: \lambda \in \Lambda\right\}$ of prime $l$-ideals of $A$ is dense if $\cap\left\{P_{\lambda}: \lambda \in \Lambda\right\}=(0)$. Clearly if $\left\{P_{\lambda}: \lambda \in \Lambda\right\}$ is a dense family of prime $l$-ideals and $a+M \leqq b+M$ for all $\lambda \in \Lambda$ then $a \leqq b$. If $A$ is a reduced $f$-ring then the family of all minimal prime $l$-ideals of $A$ is dense.

LEMMA 6. Let $A$ be a reduced $f$-ring with identity 1 . Then for an element $b>0$ in $A$ the following are equivalent:

(i). $b=\vee\{b \wedge n 1: n$ a natural number $\}$

(ii) $b$ is the join of a family of bounded elements

(iii) there is a dense family $\left\{M_{\lambda}: \lambda \in \Lambda\right\}$ of minimal prime l-ideals of $A$ such that $b+M_{\lambda}$ is bounded in $A / M_{\lambda}$, for all $\lambda \in \Lambda$.

Proof. The equivalence of (i) and (ii) is straightforward. Suppose that $b>0$ in $A$ and that $\mathcal{M}$ is the set of all minimal prime $l$-ideals $M$ of $A$ such that $b+M$ is bounded in $A / M$. In order to prove that (i) implies (iii) suppose that $I=\cap M \neq(0)$. Then $I$ contains an element $x$ with $0<x \leqq 1$. Clearly $b-x+M=b+M \geqq b \wedge n 1+M$ for all $M \in \mathcal{M}$. For every minimal prime $l$-ideal $M$ not belonging to $\mathcal{M}$ the coset $b-x+M$ is unbounded in $A / M$; for if $b-x+M \leqq n 1+M$ for some natural number $n$, then $b+M \leqq$ $x+n 1+M \leqq(n+1) 1+M$. Thus, $b-x+M>n 1+M \geqq b \wedge n 1+M$ for all minimal prime $l$-ideals $M$ of $A$ not belonging to $\mathcal{M}$, and every natural number $n$. Consequently, $b-x+M \geqq b \wedge n 1+M$ for every minimal prime $l$-ideal $M$ of $A$. As the set of all minimal prime $l$-ideals is dense, we conclude that $b-x \geqq b \wedge n 1$, and this for every natural number $n$. Thus, (i) does not hold. 
In order to prove that (iii) implies (i) suppose that $b \neq \vee\{b \wedge n 1: n$ a natural number\}. Then there is an $x>0$ in $A$ such that $b-x \geqq b \wedge n 1$ for all natural numbers $n$. For every $M \in \mathcal{M}$ we get $b-x+M \geqq b \wedge n 1+M=$ $b+M$ for some $n$, so $-x+M \geqq 0$. As on the other hand $x+M \geqq 0$, we have $0<x \in \cap \mathcal{M}$ which contradicts (iii).

We shall say that an element $b$ of a reduced $f$-ring $A$ with identity is almost-bounded if $|b|$ satisfies one of the equivalent conditions of lemma 6 . We denote the set of almost-bounded elements of $A$ by $\mathscr{E}(A)$, and from lemma 6 (iii) one readily deduces that $\mathscr{E}(A)$ is a convex sublattice and subring of $A$.

Theorem 7. Let $A$ be a reduced $f$-ring with identity and let $D: A \rightarrow A$ be a positive derivation. Then $\mathscr{E}(A) \subseteq \operatorname{Ker} D$.

Proof. By theorem 3 every minimal prime $l$-ideal of $A$ is invariant under $D$. Let $b$ be an almost-bounded element of $A$, and let $\left\{\boldsymbol{M}_{\alpha}\right\}$ be the set of minimal prime $l$-ideals of $A$ for which $b$ is bounded in $A / M_{\alpha}$. Then, for each $\alpha, D$ defines a derivation $D_{\alpha}$ on $A / M_{\alpha}$ by $D_{\alpha}\left(x+M_{\alpha}\right)=D(x)+M_{\alpha}$, and since $b^{b}$ is bounded in $A / M_{\alpha}$ we have $D_{\alpha}\left(b+M_{\alpha}\right)=0$. That is, $D(b) \in \cap_{\alpha} M_{\alpha}=(0)$.

Corollary 8. Let $A$ be a reduced $f$-ring with identity. If $y \in A$ is such that uy is almost-bounded for some $u>0$ with $u^{\perp}=(0)$, then $D(y)=0$ for every positive derivation $D: A \rightarrow A$.

Proof. By theorem 7 we have $u D(|y|)+D(u)|y|=0$ and therefore $u D(|y|)=0$, for each positive derivation $D$ on $A$. Since $A$ is reduced we then have $u \wedge D(|y|)=0$ and therefore $D(|y|)=0$ since $u^{\perp}=(0)$. Consequently $D(y)=0$.

COROLlary 9. If $A$ is a reduced $f$-ring with identity 1 such that every $x>1$ is invertible then the only positive derivation $D: A \rightarrow A$ is $D=0$.

Proof. If $A$ satisfies the assumptions then each $x>1$ has the property that $\left(x^{-1}\right)^{1}=(0)$ and $x^{-1} x=1$ is bounded. Thus $D(x)=0$ for all $x>1$. Then $D(y)=0$ for all $y \in A$, since $|y| \leqq|y| \vee 1$ for all $y \in A$.

COROLlary 10. If $D$ is a positive derivation on a totally-ordered division ring then $D=0$.

We recall that a ring $A$ is (von Neumann) regular if for each $a \in A$ there is an $x \in A$ for which $a x a=a$ and $x a x=x$. D. J. Johnson (1962) has shown that every regular $f$-ring $A$ is strongly regular, that is, for each $a \in A$ there is 
an $x \in A$ for which $a^{2} x=0$. In particular, every regular $f$-ring $A$ is reduced and $A / M$ is a totally-ordered division ring for each minimal prime $l$-ideal $M$.

THEOREM 11. If $D: A \rightarrow A$ is a positive derivation on a regular $f$-ring not necessarily with identity) then $D=0$.

Proof. Let $M$ be a minimal prime $l$-ideal of $A$. By the remarks preceding this theorem and by theorem 3 we have $D(M) \subseteq M$. The derivation $D$ defined on the totally-ordered division ring $A / M$ by $\bar{D}(x+M)=$ $D(x)+M$ then must be zero by corollary 10 . Thus, $D(A) \subseteq \cap\{M: M$ is a minimal prime $l$-ideal $\}=(0)$, since $A$ is reduced.

\section{References}

A. Bigard (1969), Contribution à la théorie des groups réticulés (Thèse, Paris).

A. Bigard and K. Keimel (1969), 'Sur les endomorphismes conservant les polaires d'un groupe réticulé archimédien', Bull. Soc. Math. France 97, 381-398.

G. Birkhoff and R. S. Pierce (1956), 'Lattice-ordered rings', Anais. Acad. Brasil Ciencias 28, 41-69.

M. Henriksen and Isbell (1962), 'Lattice-ordered rings and function rings', Pac. J. Math. 12, 533-565.

D. G. Johnson (1962), 'On a representation theorem for a class of archimedean lattice-ordered rings', Proc. Lond. Math. Soc. (3) 12, 207-225.

Ballarat Institute of Advanced Education, Ballarat.

Mathematics Department,

La Trobe University, Melbourne, Australia

and

Technische Hochschule, Darmstadt, Germany. 